\title{
Geometrically Nonlinear Aeroelastic Scaling for Very Flexible Aircraft
}

\author{
Zhiqiang Wan* \\ Beihang University, Beijing 100191, People's Republic of China \\ and \\ Carlos E. S. Cesnik \\ University of Michigan, Ann Arbor, Michigan 48109-2140 \\ DOI: $\underline{10.2514 / 1 . J 052855}$
}

\begin{abstract}
High-aspect-ratio wings present in very flexible aircraft can undergo large deformations, which results in significant changes in natural frequencies as well as in static and dynamic aeroelastic response. This geometric nonlinear behavior becomes an integral part of any aeroelastic analysis to be conducted in such class of vehicles. Aeroelastic scaling is an important way to study the aeroelastic behavior of aircraft, and it is an integral part in risk mitigation for aircraft development. However, the current aeroelastic scaling methodologies have focused on geometrically linear structures. This paper demonstrates a methodology for geometrically nonlinear aeroelastic scaling of very flexible aircraft. The known linear scaling factors and similarity rules are extended to address geometrically nonlinear aeroelastic scaling. A high-aspect-ratio flying wing in free flight is taken as an example to verify the new scaling procedure, and numerical studies are conducted using the University of Michigan's Nonlinear Aeroelastic Simulation Toolbox. Numerical results support the new approach for aeroelastic scaling of very flexible aircraft.
\end{abstract}

\begin{tabular}{ll} 
& \multicolumn{1}{c}{ Nomenclature } \\
$\overline{\boldsymbol{B}}$ & $=$ strain-displacement operator \\
$\boldsymbol{B}_{L}$ & $=$ linear strain-displacement differential operator \\
$\boldsymbol{B}_{\mathrm{NL}}(\{u\})$ & $=$ nonlinear strain-displacement operator \\
$\boldsymbol{C}_{h}$ & $=$ generalized (structural) damping matrix \\
$\boldsymbol{D}$ & $=$ material matrix \\
$\mathrm{dV}$ & $=$ domain volume \\
$\mathrm{EA}$ & $=$ beam extensional stiffness \\
$\mathrm{EI}_{\mathrm{y}}$ & $=$ out-of-plane bending stiffness \\
$\mathrm{EI}_{\mathrm{z}}$ & $=$ in-plane bending stiffness \\
$\mathrm{GJ}_{i}$ & $=$ beam torsional stiffness \\
$i$ & $=$ imaginary number \\
$\boldsymbol{K}_{\boldsymbol{K}}$ & $=$ structural stiffness matrix \\
$\boldsymbol{K}_{h}$ & $=$ generalized structural stiffness matrix \\
$\boldsymbol{K}_{L}$ & $=$ linear stiffness matrix due to small deformations \\
$\boldsymbol{K}_{\mathrm{NL}}$ & $=$ nonlinear stiffness matrix due to large \\
$\boldsymbol{K}_{T}$ & $=$ geometric deformation \\
& \\
$\boldsymbol{K}_{\sigma}$ & $=$ tangential stiffness matrix in global coordinate \\
$k_{b}$ & $=$ geometric stiffness matrix due to prestress \\
$k_{\mathrm{EA}}$ & $=$ length scaling factor \\
$k_{\mathrm{EI}}$ & $=$ bending stiffness scaling factor \\
$k_{\mathrm{GJ}}$ & $=$ torsional stiffness scaling factor \\
$k_{K}$ & $=$ stiffness scaling factor \\
$k_{L}$ & $=$ lift scaling factor
\end{tabular}

Presented as Paper 2013-1894 at the 54th AIAA/ASME/ASCE/AHS/ASC Structures, Structural Dynamics, and Materials Conference, Boston, MA, 8-11 April 2013; received 7 June 2013; revision received 15 April 2014; accepted for publication 15 April 2014; published online 17 July 2014. Copyright $\odot 2014$ by Zhiqiang Wan and Carlos E. S. Cesnik. Published by the American Institute of Aeronautics and Astronautics, Inc., with permission. Copies of this paper may be made for personal or internal use, on condition that the copier pay the $\$ 10.00$ per-copy fee to the Copyright Clearance Center, Inc., 222 Rosewood Drive, Danvers, MA 01923; include the code 1533-385X/ 14 and $\$ 10.00$ in correspondence with the CCC.

*Associate Professor, School of Aeronautic Science and Engineering; Visiting Professor, Department of Aerospace Engineering, University of Michigan, Ann Arbor, MI 48109-2140; wzq@ buaa.edu.cn. Senior Member AIAA.

${ }^{\dagger}$ Professor, Department of Aerospace Engineering, 1320 Beal Avenue 3024 FXB; cesnik@umich.edu. Fellow AIAA (Corresponding Author).

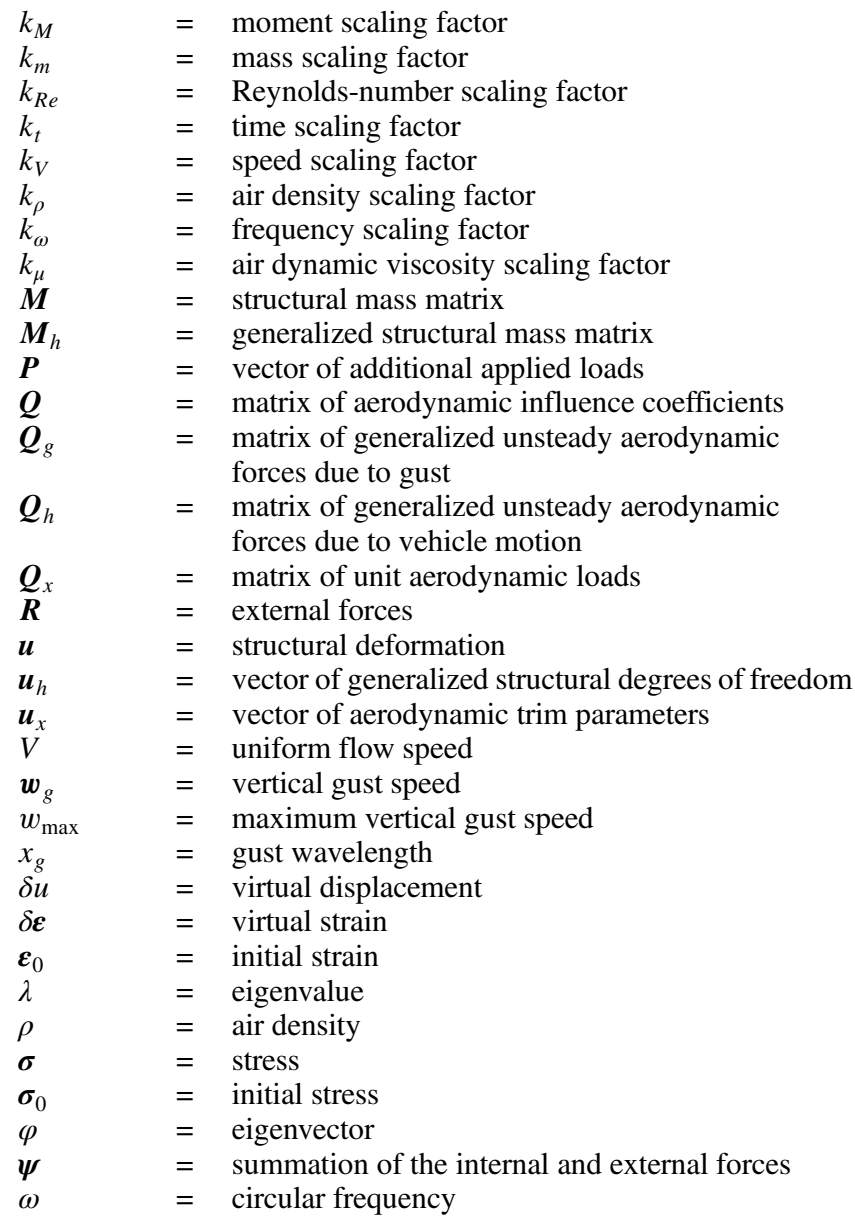

\section{Introduction}

$\mathbf{A}$ EROELASTIC scaled models for wind-tunnel testing or flight test play a key role in studying the aeroelastic characteristics of full-size aircraft, in which the aeroelastic scaling laws are the key elements [1]. The scaled models are also widely used in research studies such as active control of aeroelastic response, flutter 
characterization and mitigation, and theoretical or computational methods validation. Aeroelastic scaled models are designed and manufactured so that the results obtained from the wind-tunnel tests or flight tests can be directly related to the aeroelastic behavior of the full-size aircraft that they represent. The key elements that enable this are the aeroelastic scaling laws based upon the relationship among the physical parameters that characterize each model. Based on the use of dimensional analysis and the fundamentals of the Buckingham $\pi$ theorem, the classical approach to aeroelastic scaling was presented by Bisplinghoff et al. [2]. Since then, wind-tunnel models from aeroelastic scaling have been widely used in testing of linear structure for more than 50 years, and aeroelastic scaling considerations that enable one to relate wind-tunnel test results to the behavior of the fullsize wing or aircraft have played an important role in aircraft research and development [3] .

A comprehensive survey on aeroelastic testing for certification process and airworthiness requirement is presented in [4]. It is also discussed there the requirements for aeroelastic wind-tunnel model test and aeroelastic scaling. Wind-tunnel models are generally manufactured with a very different internal structure from the fullscale aircraft. However, it is optimized to give the design that is closest to the prespecified target elastic, inertial, and aerodynamic properties based upon some scaling parameters. The optimization enables the model to present characteristics similar to its full-scale counterpart (e.g., reduced frequencies, mode shapes, gust response, buckling limit, control surface effectiveness, etc.). An example of the process to design a flutter scaled model of a low-aspect-ratio wing using genetic algorithm is presented by $\mathrm{Wu}$ et al. [5]. Another example is the work by French and Eastep [6] that used this approach to match scaled stiffness and mass properties using five different stiffness and two different mass design approaches. Comparisons between experiment and the designs showed the effectiveness of the optimum design method for a linear, low-aspect-ratio wing. Friedmann [3] studied aeroelastic scaling for fixed- and rotary-wing aircraft and introduced a two-pronged approach that involved bringing simulation to capture the aerodynamic or control nonlinearities of the problem with the classic scaling relations. He demonstrated the aeroservoelastic scaling solution of a twodimensional airfoil combined with an actively controlled flap. For large finite-element representations, simple scaling rules were proposed by Pototzky [7] and applied to a modal formulation of the aeroservoelastic equations. That allowed the scaling of multiple discrete coordinate equations of motion from a detailed finiteelement model, and it was verified on a representation of the highspeed civil transport aircraft.

Over the past two decades, high-altitude long-endurance (HALE) aircraft have gained greater interest. These configurations present high-aspect-ratio wings for high aerodynamic performance and, combined with the low structural weight, lead to very flexible vehicles. The wings of HALE aircraft may undergo large deformations during normal operating conditions, thus exhibiting geometrically nonlinear behavior. Moreover, the low frequency response of the very flexible structure is in the same range of frequencies associated with then vehicle flight dynamics (e.g., phugoid, short period, Dutch roll, etc.), making it impossible to separate them in tests as typically done with more traditional (linear) vehicles. To understand the geometrically nonlinear aeroelastic behavior of HALE aircraft, there have been different efforts on simulation and test of fully flexible wings. Nonlinear aeroelastic solvers have been under development to improve predictions of aircraft response, stability, and overall performance, and an overview of those can be found in [8]. Among them, Cesnik et al. have developed a novel and practical solution to the nonlinear aeroelasticity and its coupling with flight dynamics of very flexible aircraft as well as built the framework for their simulation known as the University of Michigan's Nonlinear Aeroelastic Simulation Toolbox [물] . All of these studies have contributed in different ways toward the understanding of the nonlinear characteristics of highly flexible aircraft. However, due to its complex coupled nature and very limited validation data for the codes, the problem is still not fully understood.
Aeroelastic scaling in general is a good way to simulate and validate aeroelastic behavior of full-size aircraft. According to the corresponding certification process and airworthiness requirement, all numerical efforts must eventually be validated by aeroelastic scaling against experimental data so that they can be applied to new aircraft concepts. However, the literature regarding aeroelastic scaling of geometrically nonlinear structure is virtually nonexistent, and the great majority of existing scaling work focuses on linear structures. Because of the complexities of the response of very flexible structure, scaling laws for linear structure are of very limited value.

In the field of aeroelastic scaling of a geometrically nonlinear structure, [10-12] present the best attempt to nonlinear aeroelastic scaled design for a joined-wing aircraft for wind-tunnel and flight tests. This was done by first optimizing the scaled natural frequencies of the model to match the full-scale design, which is similar to the work of Miller et al. [13]. There, an optimum design of a 1/15-scaled Sensorcraft wing was achieved with equivalent tip deflection under aerodynamic loads and natural frequencies to the corresponding fullscale vehicle. This was augmented in [11] by the corresponding match of modes and then the first buckling eigenvalue. An optimization method was used for the scaled model design. The efficacy of scaling the buckling load as a means of scaling geometrically nonlinear response is examined closely in [11]. From that, it can be concluded that it is necessary to match the entire geometric stiffness matrix that depends upon internal loads of the deformed structure. More recently, [12] extended the previous two-step optimization approach by the same group to include the matching of nonlinear static deflections for designing the scaled model. Although these were steps in the right direction, there has been no fundamental aeroelastic scaling study for geometrically nonlinear structures that can be applied to generic HALE vehicles.

The objective of this paper is to present a methodology for aeroelastic scaling of geometrically nonlinear structures undergoing large deformations through theoretical demonstration and numerical verification. The proposed method is exemplified in the aeroelastic scaling of a geometrically nonlinear flying-wing aircraft in free flight.

\section{Aeroelastic Scaling Methodology}

The behavior relation between the aeroelastic scaled model to the full-size model is governed by aeroelastic scaling laws. The sections to follow present the derivations of those relations for the case of geometric linear and nonlinear structures, including the effects of the six rigid-body degrees of freedom present during free flight. Although the Buckingham $\pi$ theorem could have been used from the ground up, a direct dimensional analysis of the equations of motion was preferred in this paper. As Bisplinghoff et al. [2] showed for linear systems, the methods are equivalent, and no loss of generality is incurred in the derivation that follows.

\section{A. Basic Equations for the Linear Aeroelastic Analysis}

The basic governing equation for the aeroelastic analysis of linear structures can be represented as [14]

$$
\left(\boldsymbol{K}-\frac{1}{2} \rho V^{2} \boldsymbol{Q}\right) \boldsymbol{u}+\boldsymbol{M} \ddot{u}=\frac{1}{2} \rho V^{2} \boldsymbol{Q}_{x} \boldsymbol{u}_{x}+\boldsymbol{P}
$$

where $\boldsymbol{K}$ is the matrix of structural stiffness, $\rho$ is the air density, $V$ is the uniform flow speed, $\boldsymbol{Q}$ is the matrix of aerodynamic influence coefficients, $\boldsymbol{u}$ is the vector of structural deformation, $\boldsymbol{M}$ is the matrix of structural mass, $\boldsymbol{Q}_{x}$ is the matrix of unit aerodynamic loads, and $\boldsymbol{u}_{x}$ is the vector of aerodynamic trim parameters (e.g., angle of attack, elevator deflection, etc.), which is used to define the deflection of aerodynamic control surface and the overall rigid motion of the aircraft. Finally, $\boldsymbol{P}$ is the vector of additional applied loads. For the static aeroelastic solution and determination of the trim conditions, only the steady terms in Eq. (1) are used. It is worth noticing that, although the aerodynamic load, represented by $\boldsymbol{Q}$, is combined with the structural stiffness term in Eq. (1), it can be regarded as a complex entry with dependence on the states and its derivatives as well 
equivalent rational functions to represent wake effects. This, however, does not impact the analysis described next because the matrix will be subdivided as needed for the different aspects of the analysis.

Note that, as for geometric-nonlinear structures, the iteration of aerostructural coupling calculation should be implemented with updated geometric position considering elastic deformation, and Eq. (1) should be evaluated in a static equilibrium condition iteratively. This implies that the stiffness matrix (and not only the load vectors) in Eq. (1) should be updated for the current deformation state.

Using harmonic motion into Eq. (1), one can obtain the eigenproblem for the modal vibration analysis as [14]

$$
[\boldsymbol{K}-\lambda \boldsymbol{M})] \varphi=0
$$

Where $\lambda$ is the eigenvalue, and $\varphi$ is the corresponding eigenvector.

Note that, as for geometric-nonlinear structures, the modal analysis will depend on the structure's current state. The normal mode analysis should be performed based on the current stiffness and mass matrices, which are dependent on the current deformation state.

The equation governing the gust response analysis can be written as [14]

$$
-\omega^{2} \boldsymbol{M}_{h} u_{h}+i \omega \boldsymbol{C}_{h} u_{h}+\boldsymbol{K}_{h} u_{h}=\frac{1}{2} \rho V^{2} \boldsymbol{Q}_{h} u_{h}+\frac{1}{2} \rho V^{2} \boldsymbol{Q}_{g} \frac{w_{g}}{V}
$$

where $\omega$ is the circular frequency, $\boldsymbol{M}_{h}$ is the generalized structural mass matrix, $\boldsymbol{u}_{h}$ is the vector of generalized structural degrees of freedom (i.e., structural modes), $i$ is imaginary number, $\boldsymbol{C}_{h}$ is the generalized (structural) damping matrix, $\boldsymbol{K}_{h}$ is the generalized structural stiffness matrix, $\boldsymbol{Q}_{h}$ is the matrix of generalized unsteady aerodynamic forces due to vehicle motion, $\boldsymbol{Q}_{g}$ is the matrix of generalized unsteady aerodynamic forces due to gust, $\boldsymbol{w}_{g}$ is the vertical gust speed, and the other symbols are consistent with those in Eq. (1). The analysis of flight dynamic stability can be further attained on the basis of Eq. (3) with some transformation for stability analysis and ignoring the gust term.

A discrete vertical gust model can be applied to the vehicle on its intended flight path as shown in Fig. 1 . Without loss of generality, the shape of the gust can be the " 1 -cosine" gust defined by

$$
w_{g}=\frac{1}{2} w_{\max }\left(1-\cos \left(\frac{2 \pi x}{x_{g}}\right)\right)
$$

where $w_{\max }$ is the maximum vertical gust speed, $x_{g}$ is the gust wavelength, and $x$ is the distance that the vehicle penetrated into the gust; the gust is considered to be uniformly distributed along the span of the vehicle.

\section{B. Scaling Factors for Linear Aeroelastic Structures}

The selection of scaling factors is a critical first step of any aeroelastic scaling model design. The length scaling factor $k_{b}$, the speed scaling factor $k_{V}$, and the air density scaling factor $k_{\rho}$ are

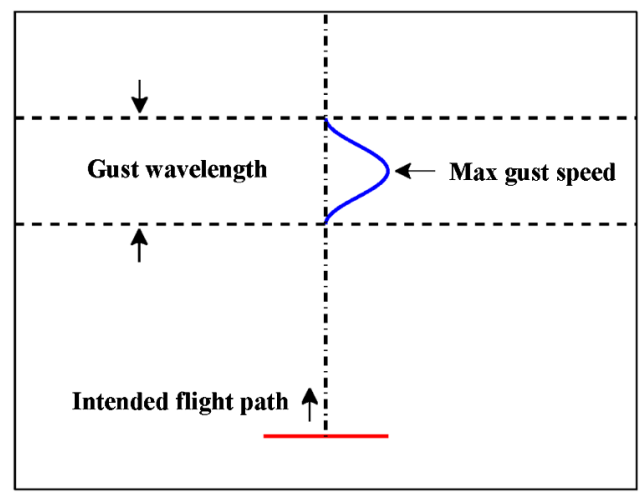

Fig. 1 "1-cosine" uniform gust profile. generally selected as the three basic scaling factors for aeroelastic model design of linear structure for wind-tunnel testing. Here, $k_{b}$ is the length ratio of the scaled model to the full-size model, $k_{V}$ is the speed ratio of the scaled model to the full-size mode, and $k_{\rho}$ is the air density ratio of the scaled model to the full-size model environment.

It is usually desirable to make aeroelastic models as large as possible for ease of fabrication and structural strength as well as to make it easier to install instrumentation in them [1]. Aeroelastic scaling models are typically larger relative to wind-tunnel test section dimensions than conventional aerodynamic models. Low-speed models may have a larger span, perhaps as large as $75 \%$ of the test section width of the wind tunnel. As for the scaled model for flight test, the length ratio is expected to be as low as possible. But it depends on the target gross takeoff weight (associated with the intended payload for instrumentation and controls) and the acceptable overall cost of the experiment.

Generally, an air density that is well up within the wind-tunnel capability is selected [1]. The larger the test density for the model, the heavier the model can be made. Usually the heavier an aeroelastic model is, the easier it is to design and fabricate. Very lightweight structures are not only fragile but are also costly to make and maintain.

For low-speed tests where compressibility effects are not important, an available wind-tunnel speed or a suitable flight speed is selected to represent some speed of the full-size model [1]. But for tests where compressibility effects are important, there must be a matched Mach number between the scaled and the full-size models so that the speed scaling factor becomes in effect the speed-of-sound scaling factor.

To meet the flutter similarity between the scaled model and the fullsize model, four similarity criteria as described next must be met on the basis of the previous three basic scaling factors [2,5]. A fifth similarity criterion must be met as an additional condition when similarity is performed on static aeroelastic response and gust response under the effect of gravity.

\section{Geometrical Similarity}

The scaling factor of geometrical similarity can be described as the aforementioned $k_{b}$. The geometrical similarity means that all size ratios of the scaled model to the full-size model, including the span ratio, chord ratio, etc., are the same as $k_{b}$. It also assumed that the scaled model and full-size model have the same airfoils.

\section{Mass Similarity}

The mass similarity criterion can be written as

$$
k_{m}=k_{\rho} k_{b}^{3}
$$

which means that the mass distribution of the scaled model should be proportional to the one of the full-size model via the mass scaling factor $k_{m}$.

\section{Stiffness Similarity}

The stiffness similarity criterion can be described as

$$
k_{K}=k_{\rho} k_{V}^{2} k_{b}
$$

which means that the stiffness distribution of the scaled model should be proportional to the one of the full-size model via the stiffness scaling factor $k_{K}$.

As for high-aspect-ratio wings, the scaling factors of bending, torsion, and extensional beam stiffness coefficients are more useful in practice and can be written, respectively, as

$$
\begin{gathered}
k_{\mathrm{EI}}=k_{K} k_{b}^{3}=k_{\rho} k_{V}^{2} k_{b}^{4} \\
k_{\mathrm{GJ}}=k_{K} k_{b}^{3}=k_{\rho} k_{V}^{2} k_{b}^{4} \\
k_{\mathrm{EA}}=k_{\mathrm{EI}} / k_{b}^{2}=k_{\rho} k_{V}^{2} k_{b}^{2}
\end{gathered}
$$


where EI is the equivalent beam (out-of-plane or in-plane) bending stiffness, GJ is the beam torsional stiffness, and EA is the beam extensional stiffness.

Based on the relation of natural frequency with mass and stiffness, the frequency scaling factor naturally follows to be

$$
k_{\omega}=\sqrt{k_{K} / k_{M}}=k_{V} / k_{b}
$$

where the time scaling factor is simply defined as

$$
k_{t}=k_{b} / k_{V}
$$

\section{Aerodynamic Similarity}

The geometrical similarity results in aerodynamic similarity when the Mach number and Reynolds number between the scaled and the full-size models are consistent, respectively. However, neither Mach number similarity nor Reynolds number similarity is easy to meet. As for low-speed flight as typical of HALE aircraft, the Mach number similarity could be ignored because of the almost incompressible air. However, the Reynolds number between the scaled and the full-size models should be the same or at least on the same order of magnitude.

The scaling factor of Reynolds number can be defined as

$$
k_{R e}=k_{\rho} k_{V} k_{b} / k_{\mu}
$$

where $k_{\mu}$ is the scaling factor for the dynamic viscosity of air.

Based on the equation for lift, the scaling factor for lift can be attained as

$$
k_{L}=k_{\rho} k_{V}^{2} k_{b}^{2}
$$

Similarly, based on the equation for aerodynamic moment, the scaling factor of moment can written as

$$
k_{M}=k_{\rho} k_{V}^{2} k_{b}^{3}
$$

\section{Froude Number Similarity}

The Froude number determines the ratio of the deflection under steady gravitational load to deflection due to aerodynamic and inertial loads. To meet similarities on static aeroelastic response and gust response, or when the gravity effect should be considered in flutter test, the Froude number between the scaled model and the fullsize model should be the same. The matching of Froude number can be satisfied by [11]

$$
k_{V}=\sqrt{k_{b}}
$$

\section{Connection Between the Scaling Factors of Linear to Geometri- cally Nonlinear Structures}

In comparison with linear structures, a geometrically nonlinear structure has its special stiffness characteristics, which depend on the current structural deformation. It is obvious that geometrical similarity, mass similarity, aerodynamic similarity, and Froude number similarity can be also met in the deformed state when the scaled model and the full-size model meet the aforementioned similarity in the undeformed state and present similar deformation in any given deformed state. Therefore, it depends on whether the stiffness of the geometrically nonlinear structure varying with structural deformation can be maintained similar between the scaled and the full-size models. This will directly impact the applicability of the linear aeroelastic scaling laws to the geometrically nonlinear structure.

Generally, the stiffness of the geometrically nonlinear structure varying with the structural deformation can be represented as [15]

$$
\left[\boldsymbol{K}_{T}\right]=\left[\boldsymbol{K}_{L}\right]+\left[\boldsymbol{K}_{\mathrm{NL}}\right]+\left[\boldsymbol{K}_{\sigma}\right]
$$

where $\left[\boldsymbol{K}_{T}\right]$ is the tangential stiffness matrix of an element in global coordinate system, $\left[\boldsymbol{K}_{L}\right]$ is the linear stiffness matrix due to small deformations, $\left[\boldsymbol{K}_{\mathrm{NL}}\right]$ is the nonlinear stiffness matrix due to large geometric deformation, and $\left[\boldsymbol{K}_{\sigma}\right]$ is the geometric stiffness matrix due to prestress.

Therefore, the $\left[\boldsymbol{K}_{T}\right]$ of the full-size model and the scaled model will be similar if it can be guaranteed that the scaling coefficients of $\left[\boldsymbol{K}_{L}\right]$, $\left[\boldsymbol{K}_{\mathrm{NL}}\right]$, and $\left[\boldsymbol{K}_{\sigma}\right]$ are the same.

\section{Correlation Between Stress and Strain}

The principle of virtual work of an element can be written as

$$
\{\delta u\}^{T}\{\boldsymbol{\psi}\}=\int\{\delta \boldsymbol{\varepsilon}\}^{T}\{\boldsymbol{\sigma}\} \mathrm{d} V-\{\delta u\}^{T}\{\boldsymbol{R}\}=0
$$

where $\{\delta u\}$ is the virtual displacement, $\{\boldsymbol{\psi}\}$ is the summation of the internal and external forces, $\{\delta \boldsymbol{\varepsilon}\}$ is the virtual strain, $\{\boldsymbol{\sigma}\}$ is the stress, $\mathrm{dV}$ is the domain volume, and $\{\boldsymbol{R}\}$ is the column vector of external forces.

The strain-displacement relation can be written in the form of strain increment as

$$
\{\delta \boldsymbol{\varepsilon}\}=[\overline{\boldsymbol{B}}]\{\delta u\}
$$

where $[\overline{\boldsymbol{B}}]$ is the differential operator that defines the straindisplacement relation.

For the problem of large displacements, the relation between strain and displacement is nonlinear; therefore, $[\overline{\boldsymbol{B}}]$ is a nonlinear function of $\{u\}$, which can be written as

$$
[\overline{\boldsymbol{B}}]=\left[\boldsymbol{B}_{L}\right]+\left[\boldsymbol{B}_{\mathrm{NL}}(\{u\})\right]
$$

where $\left[\boldsymbol{B}_{L}\right]$ is the term corresponding to the linear straindisplacement operator, and $\left[\boldsymbol{B}_{\mathrm{NL}}(\{u\})\right]$ is the additional term containing the nonlinearity in the strain-displacement relation.

Even though the problem is geometrically nonlinear, it remains materially linear. That is, the relation between stress and strain meets the linear elastic relation, which can be written as

$$
\{\boldsymbol{\sigma}\}=[\boldsymbol{D}]\left(\{\boldsymbol{\varepsilon}\}-\left\{\boldsymbol{\varepsilon}_{0}\right\}\right)+\left\{\boldsymbol{\sigma}_{0}\right\}
$$

where $[\boldsymbol{D}]$ is the material matrix, $\left\{\boldsymbol{\varepsilon}_{0}\right\}$ is the column vector of initial strains, and $\left\{\sigma_{0}\right\}$ the initial stresses.

Based on the previous relation between stress and strain, and keeping in mind that $\{\delta \varepsilon\}=\{\delta \varepsilon\}_{S}=\{\delta \varepsilon\}_{F}$, the following equations can be attained to describe the relation between the scaled model and the full-size model:

$$
\begin{gathered}
\{\delta u\}_{S}=k_{b}\{\delta u\}_{F} \\
{\left[\boldsymbol{B}_{L}\right]_{S}=\left[\boldsymbol{B}_{L}\right]_{F} / k_{b}} \\
{\left[\boldsymbol{B}_{\mathrm{NL}}\right]_{S}=\left[\boldsymbol{B}_{\mathrm{NL}}\right]_{F} / k_{b}} \\
\delta\left[\boldsymbol{B}_{\mathrm{NL}}\right]_{S}=\delta\left[\boldsymbol{B}_{\mathrm{NL}}\right]_{F} / k_{b} \\
\{\boldsymbol{\sigma}\}_{S}=k_{\rho} k_{V}^{2}\{\boldsymbol{\sigma}\}_{F} \\
{[\boldsymbol{D}]_{S}=k_{\rho} k_{V}^{2}[\boldsymbol{D}]_{F}} \\
V_{S}=k_{b}^{3} V_{F}
\end{gathered}
$$

where the subscripts $S$ and $F$ represent the scaled model and the fullsized model, respectively. 


\section{Linear Stiffness Matrix due to Small Deformation}

Based on Eqs. (17-20), the linear stiffness matrix due to small deformation is represented by

$$
\left[\boldsymbol{K}_{L}\right]=\int\left[\boldsymbol{B}_{L}\right]^{T}[\boldsymbol{D}]\left[\boldsymbol{B}_{L}\right] \mathrm{d} V
$$

For the full-size model, Eq. (28) can written as

$$
\left[\boldsymbol{K}_{L}\right]_{F}=\int\left[\boldsymbol{B}_{L}\right]_{F}^{T}[\boldsymbol{D}]_{F}\left[\boldsymbol{B}_{L}\right]_{F} \mathrm{~d} V_{F}
$$

and for the scaled model as

$$
\left[\boldsymbol{K}_{L}\right]_{S}=\int\left[\boldsymbol{B}_{L}\right]_{S}^{T}[\boldsymbol{D}]_{S}\left[\boldsymbol{B}_{L}\right]_{S} \mathrm{~d} V_{S}
$$

Substituting Eqs. (22), (26), and (27) into Eq. (무) yields

$$
\left[\boldsymbol{K}_{L}\right]_{S}=k_{\rho} k_{v}^{2} k_{b} \int\left[\boldsymbol{B}_{L}\right]_{F}^{T}[\boldsymbol{D}]_{F}\left[\boldsymbol{B}_{L}\right]_{F} \mathrm{~d} V_{F}=k_{\rho} k_{v}^{2} k_{b}\left[\boldsymbol{K}_{L}\right]_{F}
$$

and therefore the scaling factor for $\left[\boldsymbol{K}_{L}\right]$ is

$$
k_{K_{L}}=k_{\rho} k_{V}^{2} k_{b}
$$

3. Nonlinear Stiffness Matrix due to Large Geometric Deformation

Based on Eqs. (17-20), the nonlinear stiffness matrix due to large geometric deformations is given by

$$
\begin{aligned}
& {\left[\boldsymbol{K}_{\mathrm{NL}}\right]=\int\left(\left[\boldsymbol{B}_{L}\right]^{T}[\boldsymbol{D}]\left[\boldsymbol{B}_{\mathrm{NL}}\right]+\left[\boldsymbol{B}_{\mathrm{NL}}\right]^{T}[\boldsymbol{D}]\left[\boldsymbol{B}_{\mathrm{NL}}\right]\right.} \\
& \left.+\left[\boldsymbol{B}_{\mathrm{NL}}\right]^{T}[\boldsymbol{D}]\left[\boldsymbol{B}_{L}\right]\right) \mathrm{d} V
\end{aligned}
$$

For the full-size model, Eq. (33) can written as

$$
\begin{aligned}
& {\left[\boldsymbol{K}_{\mathrm{NL}}\right]_{F}=\int\left(\left[\boldsymbol{B}_{L}\right]_{F}^{T}[\boldsymbol{D}]_{F}\left[\boldsymbol{B}_{\mathrm{NL}}\right]_{F}+\left[\boldsymbol{B}_{\mathrm{NL}}\right]_{F}^{T}[\boldsymbol{D}]_{F}\left[\boldsymbol{B}_{\mathrm{NL}}\right]_{F}\right.} \\
& \left.\quad+\left[\boldsymbol{B}_{\mathrm{NL}}\right]_{F}^{T}[\boldsymbol{D}]_{F}\left[\boldsymbol{B}_{L}\right]_{F}\right) \mathrm{d} V_{F}
\end{aligned}
$$

and for the scaled model as

$$
\begin{aligned}
& {\left[\boldsymbol{K}_{\mathrm{NL}}\right]_{S}=\int\left(\left[\boldsymbol{B}_{L}\right]_{S}^{T}[\boldsymbol{D}]_{S}\left[\boldsymbol{B}_{\mathrm{NL}}\right]_{S}+\left[\boldsymbol{B}_{\mathrm{NL}}\right]_{S}^{T}[\boldsymbol{D}]_{S}\left[\boldsymbol{B}_{\mathrm{NL}}\right]_{S}\right.} \\
& \left.+\left[\boldsymbol{B}_{\mathrm{NL}}\right]_{S}^{T}[\boldsymbol{D}]_{S}\left[\boldsymbol{B}_{L}\right]_{S}\right) \mathrm{d} V_{S}
\end{aligned}
$$

Substituting Eqs. (22), (23), (26), and (27) into Eq. (프) yields

$$
\left[\boldsymbol{K}_{\mathrm{NL}}\right]_{S}=k_{\rho} k_{V}^{2} k_{b}\left[\boldsymbol{K}_{\mathrm{NL}}\right]_{F}
$$

and therefore the scaling coefficient of $\left[\boldsymbol{K}_{\mathrm{NL}}\right]$ is

$$
k_{K_{\mathrm{NL}}}=k_{\rho} k_{V}^{2} k_{b}
$$

\section{Geometric Stiffness Matrix Due to Prestress}

Based on Eqs. (17-20), the geometric stiffness matrix due to prestress satisfies the following energy relation:

$$
\{\delta u\}^{T}\left[\boldsymbol{K}_{\sigma}\right]\{u\}=\int\{\delta u\}^{T}\left[\boldsymbol{B}_{\mathrm{NL}}\right]^{T}\left\{\boldsymbol{\sigma}_{0}\right\} \mathrm{d} V
$$

For the full-size model, Eq. ( $\underline{38})$ can written as

$$
\{\delta u\}_{F}^{T}\left[\boldsymbol{K}_{\sigma}\right]_{F}\{u\}_{F}=\int\{\delta u\}_{F}^{T}\left[\boldsymbol{B}_{\mathrm{NL}}\right]_{F}^{T}\left\{\boldsymbol{\sigma}_{0}\right\}_{F} \mathrm{~d} V_{F}
$$

and for the scaled model as

$$
\{\delta u\}_{S}^{T}\left[\boldsymbol{K}_{\sigma}\right]_{S}\{u\}_{S}=\int\{\delta u\}_{S}^{T}\left[\boldsymbol{B}_{\mathrm{NL}}\right]_{S}^{T}\left\{\boldsymbol{\sigma}_{0}\right\}_{S} \mathrm{~d} V_{S}
$$

Substituting Eqs. (21), (23), (25), and (27) into Eq. (마) yields

$$
\{\delta u\}_{S}^{T}\left[\boldsymbol{K}_{\sigma}\right]_{S}\{u\}_{S}=k_{\rho} k_{V}^{2} k_{b}^{3} \int\{\delta u\}_{F}^{T}\left[\boldsymbol{B}_{\mathrm{NL}}\right]_{F}^{T}\left\{\boldsymbol{\sigma}_{0}\right\}_{F} \mathrm{~d} V_{F}
$$

Substituting Eqs. (21) and (푸) into Eq. (1), one has

$$
\begin{aligned}
& \{\delta u\}_{S}^{T}\left[\boldsymbol{K}_{\sigma}\right]_{S}\{u\}_{S}=k_{\rho} k_{V}^{2} k_{b}^{3}\{\delta u\}_{F}^{T}\left[\boldsymbol{K}_{\sigma}\right]_{F}\{u\}_{F} \\
& =k_{\rho} k_{V}^{2} k_{b}\{\delta u\}_{S}^{T}\left[\boldsymbol{K}_{\sigma}\right]_{F}\{u\}_{S}
\end{aligned}
$$

which results in

$$
\left[\boldsymbol{K}_{\sigma}\right]_{S}=k_{\rho} k_{V}^{2} k_{b}\left[\boldsymbol{K}_{\sigma}\right]_{F}
$$

Therefore, the scaling coefficient of $\left[\boldsymbol{K}_{\sigma}\right]$ is given by

$$
k_{K_{\sigma}}=k_{\rho} k_{V}^{2} k_{b}
$$

5. Condition for Aeroelastic Similarity for Geometrically Nonlinear Structures

Based on Eqs. (32), (37), and (44), one must impose similarity between the scaled model and the full-scale model for the three stiffness terms, that is

$$
k_{K}=k_{K_{L}}=k_{K_{\mathrm{NL}}}=k_{K_{\sigma}}
$$

This similarity condition along with the scaling factors for linear aeroelasticity defined by Eqs. (5-15) form the basis for the geometrically nonlinear aeroelastic scaling between two different scale models.

Therefore, the aeroelastic behavior between the scaled and the fullsize models will be similar both at the state of small deformation and in the state of large deformation, if the scaled model is designed on the basis of the aforementioned scaling factors.

\section{Numerical Verification}

To verify the aeroelastic scaling criteria for geometrically nonlinear structures, a high-aspect-ratio flying wing in free flight is used. The full-size model is based directly on the vehicle definition presented in [16].

\section{A. Model Description}

The high-aspect-ratio flying wing in free flight used as the full-size model is shown in Figs. 2 and 3. Based on that, two scaled models with different scaling factors were considered. In the analysis, all vehicles are trimmed for level flight. Flaperon control surfaces are distributed along the wing trailing edge.

The full-size vehicle has a span of $72.8 \mathrm{~m}$ and a constant chord length of $2.44 \mathrm{~m}$. The outboard one-third of the wing semispan has a dihedral angle of $10 \mathrm{deg}$. As indicated in Fig. 2, there are three pods, which are located at middle span and at two-thirds of the semispan at each side. The side pods have a mass of $6.0 \mathrm{~kg}$ each, and the center one has a mass of $27.23 \mathrm{~kg}$. The payload is applied on the center pod with a mass of $40.0 \mathrm{~kg}$.

There are two scaled models. One is scaled to match Froude number and Reynolds number where the scaled model is described as scaled model 1 . The other is scaled to only match Reynolds number and not the Froude number, where the scaled model is described as scaling model 2 . The geometric and physical properties and air parameters of the full-size model, the scaled model 1, and the scaled model 2 are listed in Tables 1,2 , respectively. For all cases, the Reynolds number is approximately 250,000 . 


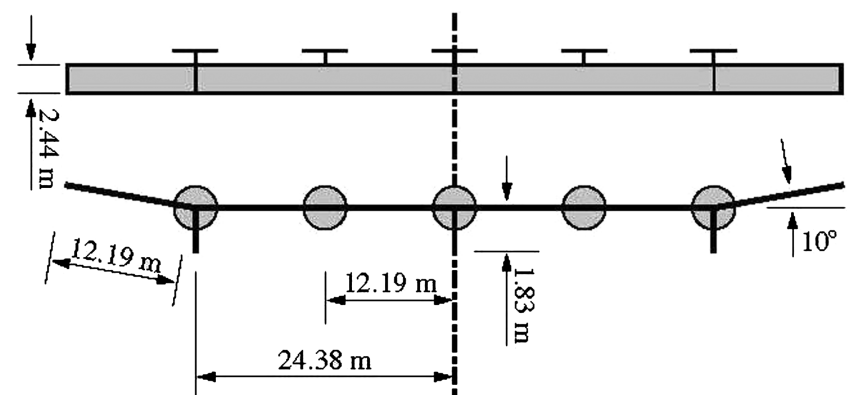

Fig. 2 Geometry of the full-scale flying-wing vehicle.

\section{B. Comparison of Results Between the Two Scaled Models and the Full-Size Vehicle}

In this section, comparison of static aeroelastic response, natural frequencies, and gust response between the full-size model and the two scaled models are investigated for the flying-wing vehicles in free flight.

\section{Trim Comparison}

Aerodynamic shape, stiffness distribution, and mass distribution have direct effect on the static aeroelastic response (vehicle deformation) as well as on trimmed parameters (angle of attack and control surface deflection). To investigate the consistence of the static aeroelastic response between the scaled models and the full-size

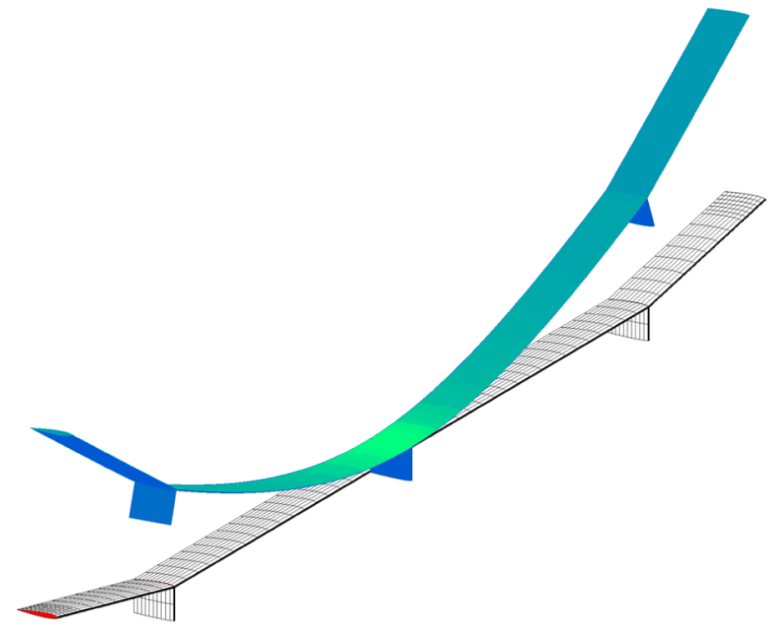

Fig. 3 Level-flight trimmed flying-wing vehicle configuration with respect to its undeformed shape.

model, the comparison of trimmed body angle of attack and flaperon angle are presented in Figs. $\underline{4}$ and 5 , respectively. The flight speed is held constant at $30 \mathrm{~m} / \mathrm{s}$ for the full-size model, and the payload on the center pod of the full-size model varies from 0 to $80 \mathrm{~kg}$. The comparison of the relative wing flatwise bending deformation normalized by the vehicle half-wingspan between the full-size model and the scaled model 1 is shown in Table $\underline{3}$, whereas the results

Table 1 Geometric properties, air parameters and scaling parameters for various flying-wing vehicle models

\begin{tabular}{|c|c|c|c|}
\hline Parameter & Full-size vehicle & Scaled model 1 & Scaled model 2 \\
\hline \multicolumn{4}{|c|}{ Geometric properties } \\
\hline Half-wingspan, $m$ & 72.8 & 14.6 & 5.97 \\
\hline Chord length, m & 2.44 & 0.488 & 0.200 \\
\hline \multicolumn{4}{|c|}{ Air parameters } \\
\hline Air density, $\mathrm{kg} / \mathrm{m}^{3}$ & 0.1096 & 1.225 & 1.225 \\
\hline Air speed $1, \mathrm{~m} / \mathrm{s}$ & 25.0 & 11.18 & 27.38 \\
\hline Air speed $2, \mathrm{~m} / \mathrm{s}$ & 30.0 & 13.42 & 32.73 \\
\hline Angle of attack & Trimmed & Trimmed & Trimmed \\
\hline$c_{l}$ & $2 \pi$ & $2 \pi$ & $2 \pi$ \\
\hline \multicolumn{4}{|c|}{ Independent scaling parameters } \\
\hline Length & & 0.200 & 0.082 \\
\hline Air density & & 11.180 & 11.180 \\
\hline Air speed & & 0.447 & 1.091 \\
\hline \multicolumn{4}{|c|}{ Dependent scaling parameters } \\
\hline$\overline{\text { Mass }}$ & & $8.94 \times 10^{-2}$ & $6.16 \times 10^{-3}$ \\
\hline $\mathrm{EI}_{y}$ & & $4.47 \times 10^{-1}$ & 1.09 \\
\hline $\mathrm{EI}_{7}$ & & $3.58 \times 10^{-3}$ & $6.01 \times 10^{-4}$ \\
\hline GJ & & $3.58 \times 10^{-3}$ & $6.01 \times 10^{-4}$ \\
\hline EA & & $8.94 \times 10^{-2}$ & $8.94 \times 10^{-2}$ \\
\hline Lift & & $8.94 \times 10^{-2}$ & $8.94 \times 10^{-2}$ \\
\hline Natural frequency & & 2.24 & 13.31 \\
\hline
\end{tabular}

Table 2 Stiffness and mass properties for various flying-wing vehicle models

\begin{tabular}{lccc}
\hline \hline \multicolumn{1}{c}{ Parameter } & Full-size vehicle & Scaled model 1 & Scaled model 2 \\
\hline Extensional stiffness EA, $\mathrm{Pa} \cdot \mathrm{m}^{2}$ & $1.00 \times 10^{10}$ & $8.94 \times 10^{8}$ & $8.94 \times 10^{8}$ \\
Torsional stiffness $\mathrm{GJ}, \mathrm{N} \cdot \mathrm{m}^{2}$ & $1.65 \times 10^{5}$ & $5.90 \times 10^{2}$ & $9.91 \times 10^{1}$ \\
Flat bending stiffness $\mathrm{EI}_{y}, \mathrm{~N} \cdot \mathrm{m}^{2}$ & $6.00 \times 10^{5}$ & $2.15 \times 10^{3}$ & $3.60 \times 10^{2}$ \\
In-plane bending stiffness EI, $\mathrm{N} \cdot \mathrm{m}^{2}$ & $1.24 \times 10^{7}$ & $4.44 \times 10^{4}$ & $7.45 \times 10^{3}$ \\
Mass per unit span $\mathrm{m}, \mathrm{kg} / \mathrm{m}$ & 2.000 & $8.944 \times 10^{-1}$ & $1.502 \times 10^{-1}$ \\
Torsional inertia $I_{x x}, \mathrm{~kg} \cdot \mathrm{m}$ & 4.150 & $7.424 \times 10^{-2}$ & $2.095 \times 10^{-3}$ \\
Flat bending inertia $I_{y y}, \mathrm{~kg} \cdot \mathrm{m}$ & $6.900 \times 10^{-1}$ & $1.234 \times 10^{-2}$ & $3.483 \times 10^{-4}$ \\
In-plane bending inertia $I_{z z}, \mathrm{~kg} \cdot \mathrm{m}$ & 3.460 & $6.189 \times 10^{-2}$ & $1.746 \times 10^{-3}$ \\
Concentrated mass at a side pod, $\mathrm{kg}$ & 6.000 & $5.366 \times 10^{-1}$ & $3.695 \times 10^{-2}$ \\
Concentrated mass at center pod, kg & 27.23 & 2.435 & $1.677 \times 10^{-1}$ \\
Concentrated payload mass, $\mathrm{kg}$ & 40.00 & 3.578 & $2.463 \times 10^{-1}$ \\
\hline \hline
\end{tabular}




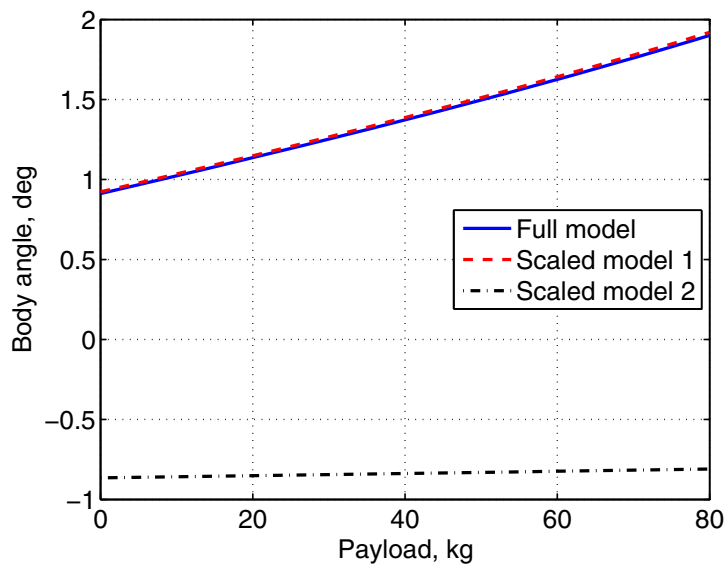

Fig. 4 Trimmed body angle of attack for different payload mass values.

between the full-size model and the scaled model 2 are shown in Table 4

As presented in Figs. 4, 5 and Tables 3, 4, the trimmed parameters and relative displacement of the scaled model matching Reynolds and Froude numbers (scaled model 1) are consistent with those of the full-size model. However, there is no consistence between the fullsize model and the scaled model 2, where only Reynolds number is matched and not Froude number.

\section{Comparison of Frequencies}

As the basis for dynamic aeroelastic response and stability, natural frequencies are very important. To investigate the comparison of natural frequencies between the full-size model and the two scaled

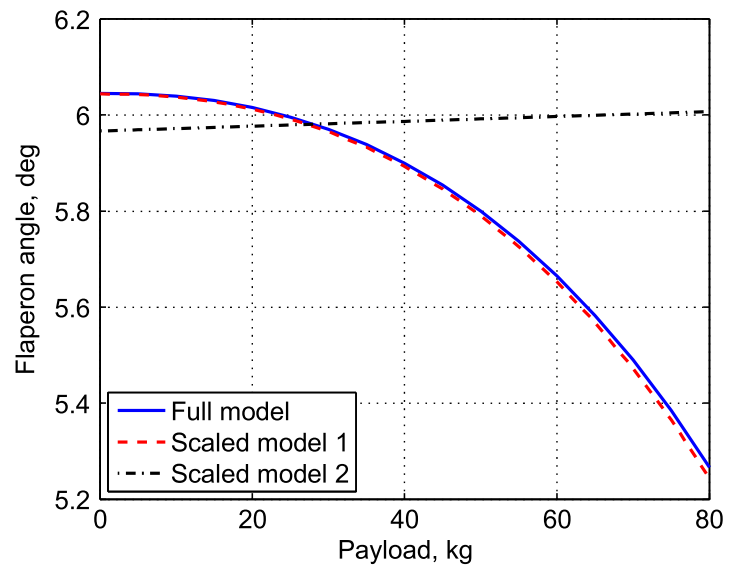

Fig. 5 Trimmed flaperon deflection for different payload mass values.

models, natural frequency analysis are carried out for both the vehicle at a given deformed state (corresponding to flight speed of $30 \mathrm{~m} / \mathrm{s}$ ) and for its undeformed state. The natural modes of the deformed structure are different from those of the undeformed structure. Table 5 presents comparison of natural frequencies between the full-size model and the scaled model 1 (matching Reynolds and Froude numbers). Table $\underline{6}$ shows comparison of natural frequencies between the full-size model and the scaled model 2 (matching Reynolds number but not Froude number).

The results indicate that natural frequencies for the scaled model 1 are consistent with those of the full-size vehicle once parameters are factored to match the units of the full-size model. However, there is no consistence between the full-size model and the scaled model 2 results.

Table 3 Comparison of relative vehicle (wing) deformation (matching Froude and Reynolds numbers)

\begin{tabular}{cccccccr}
\hline \hline & \multicolumn{3}{c}{ Airspeed 1 } & & \multicolumn{3}{c}{ Airspeed 2 } \\
\cline { 2 - 4 } Spanwise station, \% & Full-size model, \% & Scaled model 1, \% & Ratio & & Full-size model, \% & Scaled model 1, \% & Ratio \\
\hline 33.3 & 1.97 & 1.98 & 1.01 & & 1.97 & 1.98 & 1.01 \\
66.6 & 6.28 & 6.31 & 1.00 & & 6.27 & 6.30 & 1.00 \\
100 & 11.11 & 11.16 & 1.00 & 11.10 & 11.15 & 1.00 \\
\hline \hline
\end{tabular}

Table 4 Comparison of relative vehicle (wing) deformation (matching Reynolds number)

\begin{tabular}{cccccccr}
\hline \hline & \multicolumn{3}{c}{ Airspeed 1 } & & \multicolumn{3}{c}{ Airspeed 2 } \\
\cline { 2 - 3 } Spanwise station, \% & Full-size model, \% & Scaled model 2, \% & Ration & & Full-size model, \% & Scaled model 2, \% & Ratio \\
\hline 33.3 & 1.97 & 0.15 & 0.077 & & 1.97 & 0.17 & 0.086 \\
66.6 & 6.27 & 0.49 & 0.078 & & 6.27 & 0.57 & 0.091 \\
100 & 11.11 & 0.88 & 0.080 & 11.10 & 1.02 & 0.092 \\
\hline \hline
\end{tabular}

Table 5 Comparison of natural frequencies (matching Froude number and Reynolds number)

\begin{tabular}{|c|c|c|c|c|c|c|}
\hline \multirow[b]{2}{*}{ Mode } & \multicolumn{2}{|c|}{ Full-size model, $\mathrm{Hz}$} & \multicolumn{2}{|c|}{ Scaled model $1, \mathrm{~Hz}$} & \multicolumn{2}{|c|}{$\begin{array}{c}\text { Ratios (nominal } \\
k_{\omega}=2.24 \text { ) }\end{array}$} \\
\hline & Undeformed & Deformed & Undeformed & Deformed & Undeformed & Deformed \\
\hline First flat blend plus plunge & 0.30 & 0.29 & 0.66 & 0.66 & 2.24 & 2.24 \\
\hline First flat bend plus roll & 0.64 & 0.63 & 1.42 & 1.42 & 2.24 & 2.24 \\
\hline First torsion plus yaw & 1.25 & 1.14 & 2.79 & 2.54 & 2.24 & 2.24 \\
\hline First edge bed plus lead-lag plus pitch & 1.52 & - & 3.40 & - & 2.24 & - \\
\hline Second flat bend plus plunge & 1.60 & 1.59 & 3.58 & 3.57 & 2.24 & 2.24 \\
\hline Second flat bend plus roll & 1.61 & 1.60 & 3.60 & 3.58 & 2.24 & 2.24 \\
\hline Second torsion plus lead-lag plus pitch & - & 1.85 & - & 4.14 & - & 2.24 \\
\hline Second torsion plus pitch & 2.37 & - & 5.31 & - & 2.24 & - \\
\hline Edge bend plus lead-lag plus pitch & - & 2.66 & - & 5.95 & - & 2.24 \\
\hline Second torsion plus first edge bend plus yaw & 2.97 & 2.96 & 6.64 & 6.61 & 2.24 & 2.24 \\
\hline Third flat bend plus roll & 4.10 & 4.10 & 9.17 & 9.17 & 2.24 & 2.24 \\
\hline
\end{tabular}


Table 6 Comparison of natural frequencies (matching Reynolds number only)

\begin{tabular}{|c|c|c|c|c|c|c|}
\hline \multirow[b]{2}{*}{ Mode } & \multicolumn{2}{|c|}{ Full-size model, $\mathrm{Hz}$} & \multicolumn{2}{|c|}{ Scaled model $2, \mathrm{~Hz}$} & \multicolumn{2}{|c|}{$\begin{array}{c}\text { Ratios (nominal } \\
k_{\omega}=13.31 \text { ) }\end{array}$} \\
\hline & Undeformed & Deformed & Undeformed & Deformed & Undeformed & Deformed \\
\hline First flat blend plus plunge & 0.30 & 0.29 & 3.93 & 3.93 & 13.30 & 13.37 \\
\hline First flat bend plus roll & 0.64 & 0.63 & 8.46 & 8.46 & 13.30 & 13.33 \\
\hline First torsion plus yaw & 1.25 & 1.14 & 16.62 & 16.50 & 13.31 & 14.53 \\
\hline First edge bed plus lead-lag plus pitch & 1.52 & - & 20.24 & - & 13.31 & - \\
\hline Second flat bend plus plunge & 1.60 & 1.59 & 21.31 & 21.02 & 13.30 & 13.18 \\
\hline Second flat bend plus roll & 1.61 & 1.60 & 21.39 & 21.30 & 13.30 & 13.32 \\
\hline Second torsion plus lead-lag plus pitch & - & 1.85 & - & 21.38 & - & 11.54 \\
\hline Second torsion plus pitch & 2.37 & - & 31.59 & - & 13.31 & - \\
\hline Edge bend plus lead-lag plus pitch & - & 2.66 & - & 31.71 & - & 11.93 \\
\hline Second torsion plus first edge bend plus yaw & 2.97 & 2.96 & 39.51 & 39.77 & 13.31 & 13.44 \\
\hline Third flat bend plus roll & 4.10 & 4.10 & 54.48 & 54.49 & 13.30 & 13.30 \\
\hline
\end{tabular}

\section{Stability Comparison}

To assess the vehicle stability, a linearization of the aeroelastic equations of motion at each trimmed condition is performed for the full-size model and the scaled models 1 and 2 for different payload values. Then the longitudinal flight dynamic modes are evaluated for their stability. Figure 6 shows the root loci of the phugoid mode of the three models, where the results for the scaled models have already been factored by their corresponding frequency ratios. The flight speed of $30 \mathrm{~m} / \mathrm{s}$ is kept constant for the full-size model while the payload varies (solid symbols standing for the zero payload cases).

As shown in Fig. 6, the root loci of the phugoid mode for the scaled model 1 (matching Froude and Reynolds numbers) is consistent with

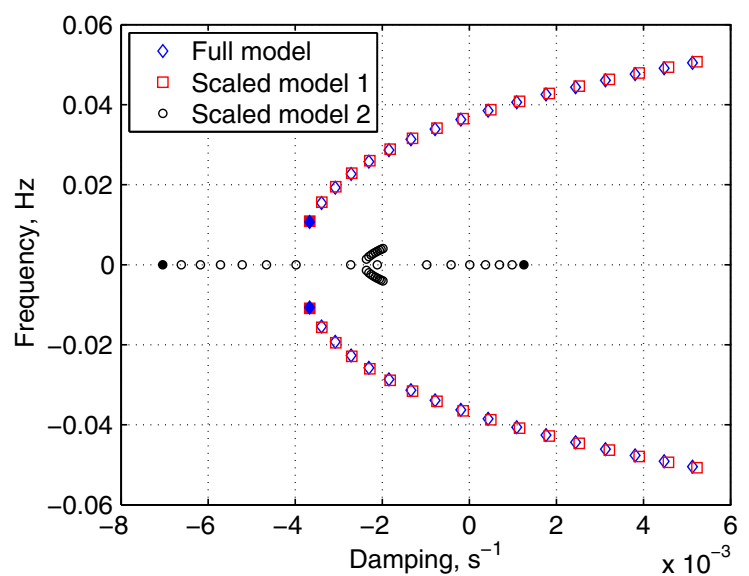

Fig. 6 Phugoid mode comparison between the full-size model and the two scaled models. the one of the full-size vehicle at different values of payload (after parameters are factored to match the units of the full-size model). However, the same does not hold for scaled model 2, indicating once again the importance of enforcing the Froude number similarity as well.

\section{Comparison of Gust Response}

To understand the consistence of gust response between the fullsize model and scaled models 1 and 2, the discrete gust model described previously is used in the fully nonlinear, time-marching solution. The wavelength is equivalent to 25 times the vehicle chord length, which corresponds to $61 \mathrm{~m}$ for the full-size model. The maximum vertical gust speed is $10 \mathrm{~m} / \mathrm{s}$ for full-size model, while the vehicle is flying at $30 \mathrm{~m} / \mathrm{s}$. The gust excitation is applied for the fullsize model after $0.1 \mathrm{~s}$ of flying trimmed in calm air (no change in trim parameters while going through gust). The gust parameters for the scaled models have been properly scaled according to the scaling factors in Table 1.

The longitudinal and vertical displacements of the origin of the body frame (center span, at the beam reference line position) are plotted in Fig. 7 for the full-size model and the scaled models 1 and 2 . The comparisons for body pitch angle and wing-tip displacements are shown in Figs. 8 and 9, respectively. Finally, flatwise bending and torsional moments at the vehicle midspan are also plotted in Figs. 10 and 11 , respectively. For all these results, the results from the scaled models have been scaled to match the units of the full-size model.

From those plots, the body-frame displacements, the body pitch angle, the relative wing-tip displacement, and the flatwise bending moment and torsional moment at the center of the vehicles for the scaled model 1 are consistent with those of the full-size model (after results are factored to match the units of the full-size model). Once again, there is no consistence between the full-size model results and

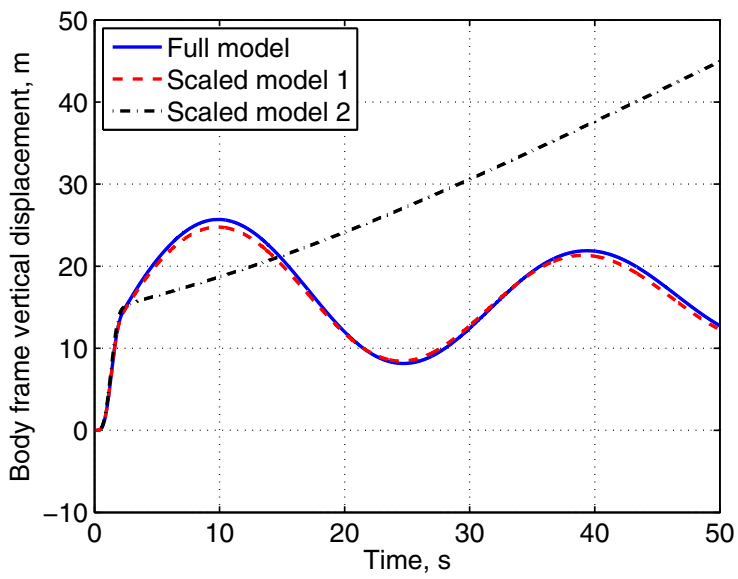

b) Vertical displacement 


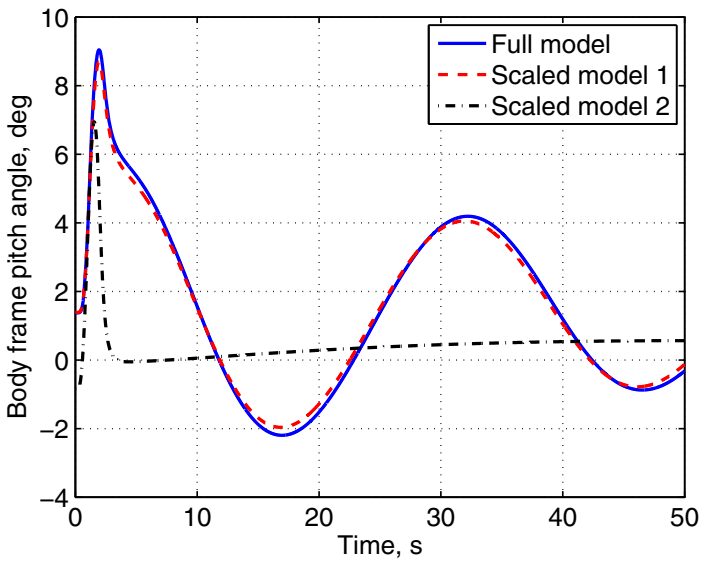

Fig. 8 Body pitch angle response due to gust.

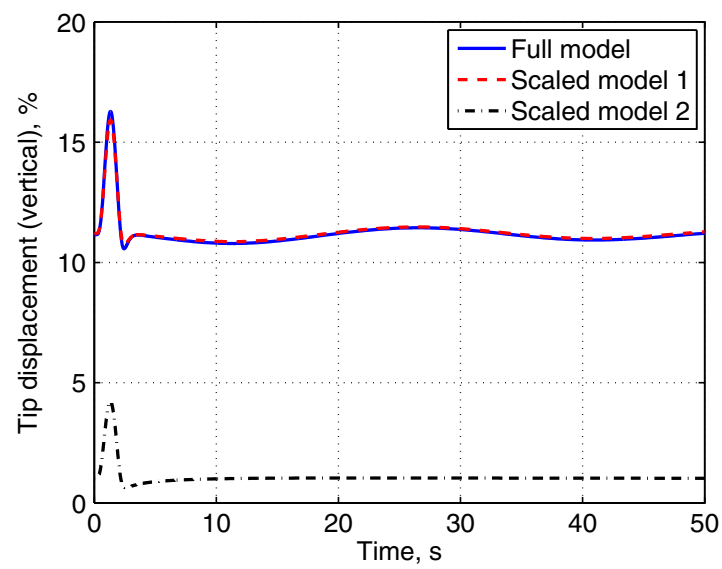

Fig. 9 Relative tip wing displacement with respect to vehicle half-span response due to gust.

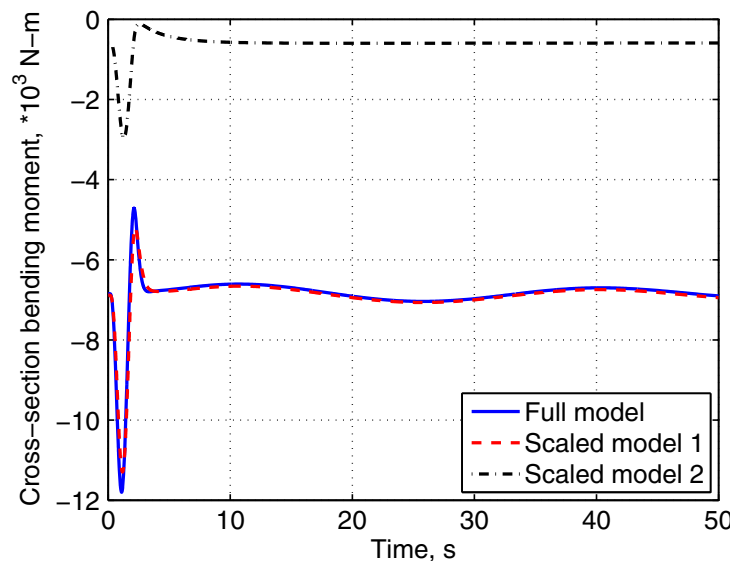

Fig. 10 Bending moment at the vehicle center response due to gust.

the scaled model, which only matches Reynolds number but does not match Froude number.

\section{Conclusions}

An aeroelastic scaling methodology for (geometrically nonlinear) very flexible aircraft has been presented. Based on theoretical derivation and numerical verification on a flying-wing vehicle in free flight, the aeroelastic scaling laws for linear structure were extended to address geometrically nonlinear structures. A nonlinear aeroelastic analysis including static aeroelastic response, gust response, and frequencies have been conducted on the full-size model and two

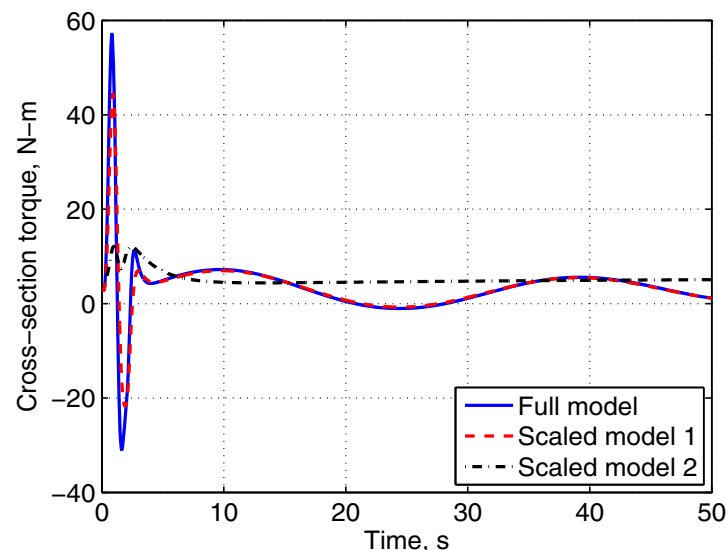

Fig. 11 Torsional moment at the vehicle center response due to gust.

sample scaled models using the University of Michigan's Nonlinear Aeroelastic Simulation Toolbox.

The analysis results indicate that the current existing aeroelastic scaling law for linear structure is also suitable for geometrically nonlinear structure with large deformation. It was shown that the scaling factors for the linear and nonlinear parts of the stiffness matrix follow the same similarity rule. Moreover, it is imperative that the Froude number similarity be met. The Froude number determines the ratio of the deflection under gravitational load to deflection due to aerodynamic and inertial loads. Satisfying the Froude number similarity guarantees that the large static wing deflection under initial trim condition is met, and therefore the nonlinear stiffness properties between models is satisfied as well. The stiffness has a direct impact into the static and dynamic aeroelastic response of the system. Reynolds-number similarity is also important particularly when its value is low enough for laminar/turbulence transition. In practice, however, matching the Reynolds number criterion may present a challenge in view of the other scaling factors.

\section{Acknowledgments}

The authors are grateful to Weihua Su (University of Michigan, currently assistant professor at the University of Alabama) for supporting the numerical validation. The work presented here was developed while the first author was spending his sabbatical at the University of Michigan, which was sponsored by the China Scholarship Council and the National Natural Science Foundation of China (grants 11172025 and 91116005).

\section{References}

[1] Busan, R., "Flutter Model Technology," Wright-Patterson Air Force Base WL-TR-97-3074, OH, Jan. 1998.

[2] Bisplinghoff, R. L., Ashley, H., and Halfman, R. L., "Aeroelasticity," Addison-Wesley, Cambridge, MA, 1955, pp. 695-716.

[3] Friedmann, P. P., "Aeroelastic Scaling for Fixed and Rotary-Wing Aircraft with Application," 41st AIAA/ASME/ASCE/ AHS/ASC Structures, Structural Dynamics, and Materials Conference and Exhibit, AIAA Paper 2000-1688, April 2000.

[4] Cooper, J., Lind, R., and Wright, J., "Aeroelastic Testing and Certification," Encyclopedia of Aerospace Engineering, edited by Blockley, Richard, and Shyy, Wei, John Wiley and Sons Ltd., West Sussex, Vol. 3, 2010, pp. 1641-1651, Chap. 138.

[5] Wu, Q., Wan, Z., and Yang, C., "Design Optimization of Scaled Flutter Model Considering Structural Dynamics and Flutter Constraints," Chinese Acta Aeronautica et Astronautica Sinica, Vol. 32, No. 7, 2011, pp. 1210-1216.

[6] French, M., and Eastep, F., "Aeroelastic Model Design Using Structural Optimization," 4th Symposium on Multidisciplinary Analysis and Optimization, AIAA Paper 1992-4730, Sept. 1992.

[7] Pototzky, A. S., "Scaling Laws Applied to a Modal Formulation of the Aeroservoelastic Equations," 43rd AIAA/ASME/ ASCE/AHS/ASC Structures, Structural Dynamics, and Materials Conference, AIAA Paper 2002-1598, April 2002. 
[8] Cesnik, C. E. S., Senatore, P. J., Su, W., Atkins, E. M., and Shearer, C. M., "X-HALE: A Very Flexible UAV for Nonlinear Aeroelastic Tests," AIAA Journal, Vol. 50, No. 12, Dec. 2012, pp. 2820-2833. doi:10.2514/1.J051392

[9] Shearer, C. M., and Cesnik, C. E. S., "Nonlinear Flight Dynamics of Very Flexible Aircraft," Journal of Aircraft, Vol. 44, No. 5, 2007, pp. $1528-1545$.

[10] Blair, M., Garmann, D., Canfield, R., Bond, V., Pereira, P., and Suleman, A., "Nonlinear Aeroelastic Scaling of a Joined-Wing Concept," 48th AIAA/ASME/ASCE/AHS/ASC Structures, Structural Dynamics, and Materials Conference, AIAA Paper 2007-1887, April 2007.

[11] Bond, V. L., Canfield, R. A., Suleman, A., and Blair, M., "Aeroelastic Scaling of a Joined Wing for Nonlinear Geometric Stiffness," AIAA Journal, Vol. 50, No. 3, 2012, pp. 513-522.

[12] Ricciardi, A. P., Canfield, R. A., Patil, M. J., and Lindsey, N., "Nonlinear Aeroelastic Scaling of a Joined-Wing Aircraft," 53rd AIAA/ASME/
ASCE/AHS/ASC Structures, Structural Dynamics and Materials Conference, AIAA Paper 2012-1454, April 2012.

[13] Miller, S., Vio, G., Cooper, J., and Sensburg, O., "Optimization of a Scaled Sensorcraft Model with Passive Gust Alleviation," 12th AIAA/ ISSMO Multidisciplinary Analysis and Optimization Conference, AIAA Paper 2008-5875, Sept. 2008.

[14] Rodden, W. P., and Johnson, E. H., "MSC/Nastran Aeroelastic Analysis User's Guide v.68," MSC Software Corp., Los Angeles, CA, 1994.

[15] Bathe, K.-J., Finite Element Procedures, Prentice-Hall, Upper Saddle River, NJ, 1996, pp. 540-568.

[16] Su, W., and Cesnik, C. E. S., "Dynamic Response of Highly Flexible Flying Wings," AIAA Journal, Vol. 49, No. 2, 2011, pp. 324-339. doi: $10.2514 / 1 . J 050496$
W. Silva Associate Editor 